\title{
Palmar Digital Neuropathy With Anatomical Variation of Median Nerve: Usefulness of Orthodromic Technique: A Case Report
}

\author{
Jun Soo Noh, MD' ${ }^{1}$ Jong Woong Park, MD, PhD², Hee-Kyu Kwon, MD, PhD ${ }^{1}$
}

Departments of ${ }^{1}$ Physical Medicine and Rehabilitation, ${ }^{2}$ Orthopedic Surgery, Korea University Anam Hospital, Seoul, Korea

\begin{abstract}
Anatomic variation of palmar digital nerve pathways were reported in several cases. Selective exploration of palmar digital nerves with a nerve conduction study has been challenging, because of technical issues. We report a patient who received bilateral carpal tunnel release operation, complaining of a tingling sensation, and hypoesthesia on the middle and ring fingers. An electrodiagnostic study revealed a sensory neuropathy of palmar digital nerve of the left median nerve, supplying the ulnar side of the middle finger, and radial side of the ring finger. She underwent re-operation of open left carpal tunnel release, and a branching site of common digital nerves of the median nerve was identified not at the palm, but at a far proximal site around the distal wrist crease. Usefulness of an orthodromic sensory conduction study was clarified to eliminate volume conducted response or co-activation of nearby nerves in the patient with selective involvement of palmar digital nerve.
\end{abstract}

Keywords Median nerve, Electrodiagnosis, Carpal tunnel syndrome, Median neuropathy

\section{INTRODUCTION}

As the median nerve passes under the flexor retinaculum it enlarges, flattens, and usually divides into two portions: lateral and medial. The lateral portion then divides into 3 proper palmar digital nerves, in which 2 of them supply the sides of the thumb, and one supplies the radial side of the index finger. The medial portion of median nerve divides into 2 common palmar digital branches, after passing through the transverse carpal ligament. Each common palmar digital nerve runs towards the cleft between the index and middle fingers, and the middle and ring fingers. Then they split into 2 proper digital nerves, to the adjoining sides of the 2nd to 4 th digits [1-3].

Selective exploration of palmar digital nerves with a nerve conduction study (NCS) has been challenging, because of technical issues such as innervation overlaps, volume conducted responses, and inadvertent activation of nearby nerves [2]. In our study, we report a rare variation of the course of the common palmar digital nerves of

Received June 14, 2018; Accepted August 21, 2018

Corresponding author: Hee-Kyu Kwon

Department of Physical Medicine and Rehabilitation, Korea University Anam Hospital, 73 Inchon-ro, Seongbuk-gu, Seoul 02841, Korea. Tel: +82-2-9206471, Fax: +82-2-929-9951, E-mail: hkkwon@korea.ac.kr

ORCID: Jun Soo Noh (http://orcid.org/0000-0002-4100-9154); Jong Woong Park (http://orcid.org/0000-0003-2751-2519); Hee-Kyu Kwon (http://orcid. org/0000-0002-6230-2907).

(c) This is an open-access article distributed under the terms of the Creative Commons Attribution Non-Commercial License (http://creativecommons.org/ licenses/by-nc/4.0) which permits unrestricted noncommercial use, distribution, and reproduction in any medium, provided the original work is properly cited. Copyright $\odot 2019$ by Korean Academy of Rehabilitation Medicine 
the median nerve, and its neuropathy. We also describe how we identified palmar digital neuropathy with an orthodromic sensory conduction study.

\section{CASE REPORT}

A 33-year-old healthy woman with a 1-year history of progressive sensory change on the left hand, visited the Department of Physical Medicine and Rehabilitation August 30, 2017. She had received bilateral open carpal tunnel release operation May 24, 2017, at a private orthopedic clinic. Tingling sensation and hypoesthesia of the thumb and index finger were relieved after surgery, but symptoms of the middle and ring fingers persisted and were aggravated. On physical examination, all deep tendon reflexes were normoactive in bilateral upper extremities, and no muscle wasting was noted including hand intrinsic muscles. Muscle strength was normal in bilateral upper extremities, including thumb abduction
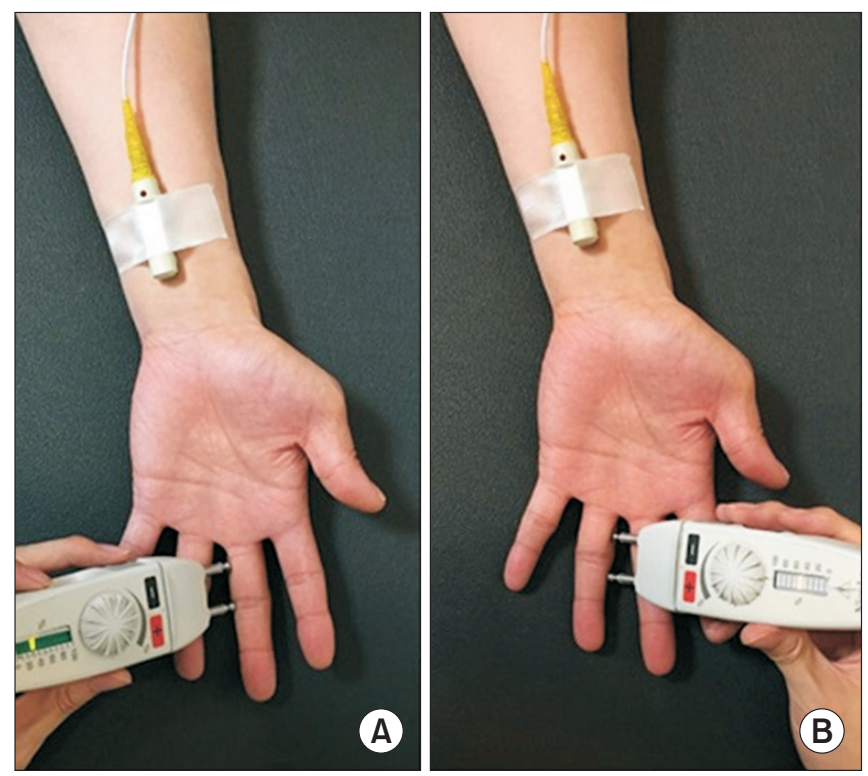

Fig. 1. Orthodromic sensory conduction study of palmar digital nerve, supplying the ulnar side of the middle finger (A) and radial side of the ring finger (B). and opposition. Hypoesthesia was noted on the ulnar

Table 1. Motor and sensory nerve conduction study

\begin{tabular}{|c|c|c|c|c|c|c|c|}
\hline & Stimulation site & Recording site & $\begin{array}{c}\text { Latency } \\
\text { (ms) }\end{array}$ & Amplitude & $\begin{array}{l}\text { Distance } \\
\text { (cm) }\end{array}$ & $\begin{array}{l}\text { NCV } \\
(\mathrm{m} / \mathrm{s})\end{array}$ & $\begin{array}{c}\text { F wave } \\
\text { (ms) }\end{array}$ \\
\hline \multicolumn{8}{|l|}{ Motor } \\
\hline Rt. median & Wrist & APB & 3.5 & 11.3 & 19.5 & 59 & 25.5 \\
\hline \multirow[t]{3}{*}{ Rt. ulnar } & Wrist & $\mathrm{ADM}$ & 3.0 & 16.6 & 15.5 & 53 & 25.5 \\
\hline & Below elbow & $\mathrm{ADM}$ & 5.9 & 16.3 & 10.0 & 56 & \\
\hline & Above elbow & $\mathrm{ADM}$ & 7.7 & 16.2 & & & \\
\hline \multicolumn{8}{|l|}{ Sensory } \\
\hline \multirow[t]{6}{*}{ Lt. median } & Wrist & Thumb & $2.0 / 2.5$ & 56 & 10 & - & - \\
\hline & Wrist & II digit & $2.5 / 3.3$ & 41 & 14 & - & - \\
\hline & Wrist & III digit & $2.5 / 3.1$ & $19^{*}$ & 14 & - & - \\
\hline & III digit (radial side) & Wrist & $3.2 / 3.5$ & 19 & 14 & - & - \\
\hline & III digit (ulnar side) & Wrist & - & $\mathrm{NR}^{*}$ & - & - & - \\
\hline & IV digit (radial side) & Wrist & - & $\mathrm{NR}^{*}$ & - & - & - \\
\hline \multirow[t]{2}{*}{ Lt. ulnar } & Wrist & IV digit (ulnar side) & $2.3 / 3.2$ & 37 & 14 & - & - \\
\hline & Wrist & V digit & 2.3/3.1 & 54 & 14 & - & - \\
\hline \multirow[t]{4}{*}{ Rt. median } & Wrist & III digit & $2.8 / 3.4$ & 40 & 14 & - & - \\
\hline & III digit (radial side) & Wrist & $3.1 / 3.6$ & 18 & 14 & - & - \\
\hline & III digit (ulnar side) & Wrist & $3.1 / 3.7$ & 18 & 14 & - & - \\
\hline & IV digit (radial side) & Wrist & $3.2 / 3.6$ & 17 & 14 & - & - \\
\hline Rt. ulnar & Wrist & IV digit (ulnar side) & $2.2 / 2.9$ & 29 & 14 & - & - \\
\hline
\end{tabular}

Latency of sensory nerve is divided into onset/peak. Amplitudes are measured in millivolt ( $\mathrm{mV}$, motor nerve) and in microvolt $\left(\mu \mathrm{V}\right.$, sensory nerve). Abnormal values are represented with asterisk $\left(^{*}\right)$.

$\mathrm{NCV}$, nerve conduction velocity; APB, abductor pollicis brevis; ADM, abductor digiti minimi; NR, no response. 
side of middle finger, and the radial side of ring finger, on the palmar side of the left hand. Tinel's sign was positive, with proximal wrist crease tapping.

An electrodiagnostic study was performed September 5 , 2017. On NCS, left median compound muscle action potential was within normal range. Left antidromic median sensory response with the middle finger recording was of low amplitude, compared to the sound side. To eliminate the possibility of volume conducted response, the orthodromic sensory conduction study was performed (Fig. 1). Orthodromically conducted sensory response was unobtainable at the wrist with ulnar side of middle finger and radial side of ring finger stimulation. But the response was normal, with the radial side of middle finger stimulation (Table 1, Fig. 2). Needle electromyogram revealed large amplitude and long duration motor unit action potential, with reduced recruitment patterns in the left abductor pollicis brevis muscle. Normal motor unit potential was observed in all other muscles examined (Supplementary Table S1). The patient was diagnosed with sensory neuropathy of palmar digital nerve of the left median nerve, supplying the ulnar side of the middle finger and radial side of the ring finger.

Ultrasonography was performed on the same day as the electrodiagnostic study. No abnormal findings such as nerve swelling and impingement were found at the palm and carpal tunnel. However, compression and swelling of the median nerve with fluid collection were noted at the distal wrist crease. Considering the above findings, com- pression of common digital branch of the median nerve at distal wrist crease was suspected (Supplementary Fig. S1).

The patient underwent re-operation of open left carpal tunnel release October 26, 2017. Interestingly, a branching site of common digital nerves of the median nerve was identified not at the palm, but at a far proximal site around the distal wrist crease (Fig. 3). In addition, a traumatic neuroma approximately $0.5 \times 0.5 \mathrm{~cm}$ was identified,
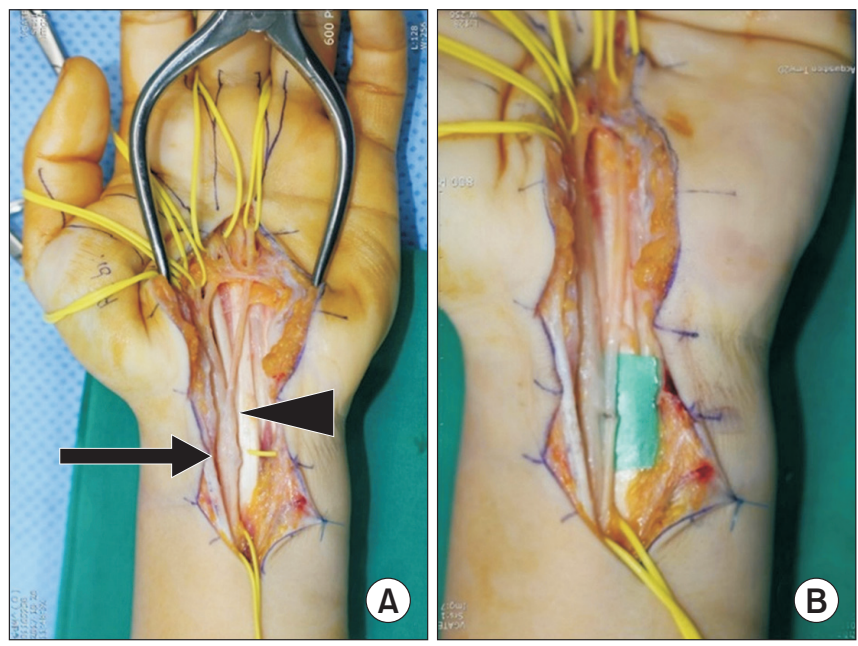

Fig. 3. Divergence of common palmar digital nerves (arrowhead) was found, around a distal wrist crease level. A traumatic neuroma $(0.5 \times 0.5 \mathrm{~cm}$, arrow) was identified, proximal to a diverging site (A). Neuroma excision and microscope-assisted to direct the end-to-end neurorrhaphy were performed (B).
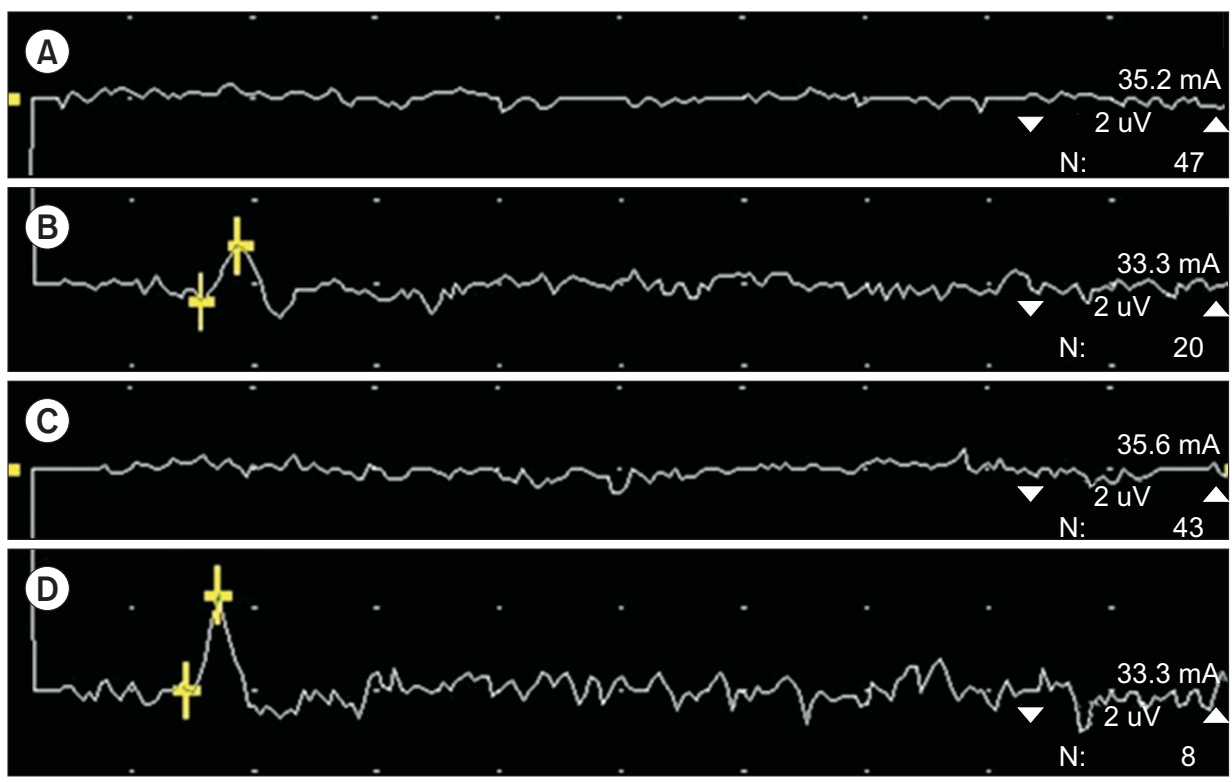

Fig. 2. Orthodromic median sensory response was not obtainable with the left ulnar side of III digit and the radial side of IV digit stimulations, in preoperative study (A and C), but obtainable with low amplitude postoperatively (B and D). 
Table 2. Sensory nerve conduction study (follow-up)

\begin{tabular}{|c|c|c|c|c|c|c|}
\hline & \multirow{2}{*}{ Stimulation site } & \multirow{2}{*}{ Recording site } & \multicolumn{2}{|c|}{ Latency (ms) } & \multirow{2}{*}{$\begin{array}{l}\text { Amplitude } \\
(\mathbf{u V})\end{array}$} & \multirow{2}{*}{$\begin{array}{c}\text { Distance } \\
\text { (cm) }\end{array}$} \\
\hline & & & Onset & Peak & & \\
\hline \multirow[t]{4}{*}{ Lt. median } & Wrist & III digit & 2.9 & 3.4 & $11^{*}$ & 14 \\
\hline & III digit (radial side) & Wrist & 2.2 & 3.0 & $6^{*}$ & 14 \\
\hline & III digit (ulnar side) & Wrist & 3.1 & 3.7 & $1^{*}$ & 14 \\
\hline & IV digit (radial side) & Wrist & 2.9 & 3.4 & $2^{*}$ & 14 \\
\hline
\end{tabular}

Abnormal values are represented with asterisk $\left({ }^{*}\right)$.

on just the proximal site to the divergence of the median nerve. Neuroma excision and microscope-assisted direct end to end neurorrhaphy were performed. Severe tenosynovitis around flexor tendons at the left carpal tunnel was also identified, and tenosynovectomy of flexor tendons was performed.

Six months after surgery, pain on the left middle and ring fingers reduced by $70 \%$. A follow-up electrodiagnostic study was performed April 10, 2018 (Table 2, Fig. 2). Previously unobtainable orthodromically conducted sensory responses, were detectable with low amplitudes. Aside from that, the left median sensory nerve action potentials (SNAPs) decreased in antidromic response with the middle finger recording, and orthodromically recorded sensory response in the radial side of the middle finger.

\section{DISCUSSION}

Median nerve variations are not uncommon $[4,5]$. These features are one of the reasons for atypical findings clinically and electrophysiologically, and can lead to diagnostic errors. Focal neuropathies and ischemic neuropathies can occur at any location. Therefore, clinicians should be aware of the anatomy of nerve structure, as well as its variations. Normally, median nerve lies between tendons of flexor carpi radialis and palmaris longus, proximal to flexor retinaculum [5]. Anatomic variations of palmar digital nerve pathways were reported in several cases. Usually, palmar digital nerves do not branch out, until the median nerve passes through the flexor retinaculum. Distal to the retinaculum, the nerve expands, and typically divides into 5 branches. Lanz [5] reported cases of median nerve divergence at far proximal parts, divided by the median artery. When the median nerve divides, the diameter of each part is reported variably: equal, radial dominant, or ulnar dominant. Schultz et al. [6] also found a far proximal division of the median nerve, with an accessory lumbrical muscle, between the 2 common palmar digital nerves. In our case, the diverging site was found at the distal wrist crease level, and it was neither divided by a vessel, nor muscle. The radial part supplying the 2nd to 3rd fingers, was thicker than the ulnar part supplying 3rd to 4 th fingers. Also, the neuroma was found just proximal, to the divergence point. This neuroma was probably formed due to an incomplete decompression of previous carpal tunnel release surgery, and is most likely the culprit responsible for the patient's hypoesthesia on the 3rd web space, and adjoining fingers. Recognition of possibilities of anatomical variations in common palmar digital nerves can enable us to recognize unexpected symptoms and signs which can occur in medical practice.

An orthodromic conduction study of the median nerve is widely performed in electrodiagnostic study, and has the following advantages: nerves that are stimulated in orthodromic technique are sensory, but those in antidromic technique are mixed. Thus, an occasional motor artifact possibly interfering with a sensory response occurs less [7]. Also, near-nerve recordings are better suited for the orthodromic, because inadvertent activation of a neighboring nerve is less common with orthodromic stimuli, than with antidromic stimuli. Therefore, eventual selective impairment of the palmar digital nerve in this case was better demonstrated with orthodromic sensory conduction studies.

Complications after carpal tunnel release surgery are rare $(0.19 \%-0.49 \%)$ [8]. However, if the symptom persists, there are 2 possibilities: incomplete carpal tunnel release, or other associated musculoskeletal problems. Since it is difficult to quantify subjective symptoms and physical examination findings after carpal tunnel release surgery, objective measurements that can quantify symptom improvement are needed. Latency and amplitude of SNAP are possible sensitive parameters in that they 
started to show improvement 2 weeks after surgery, and persisted until 3 months postoperatively [9]. Additionally, ultrasonography may be beneficial in evaluating postoperative changes, and other possibly combined musculoskeletal problems. An ultrasonographic cross-sectional area (CSA) measured at the site of maximal median nerve enlargement, and $2 \mathrm{~cm}$ proximal from the distal wrist crease (DWC) in the forearm decreased 3 weeks after surgery [10]. Also, the ratio of CSA of maximum median nerve enlargement to that of the forearm showed correlation with electrophysiological findings, although the extent of decrement showed no correlation with symptom severity [10]. In short, physicians can choose appropriate NCS or ultrasonography in patients with persistent or residual symptoms, to objectively assess postoperative changes, and eventually practice proper management.

In this patient, while the previously unobtainable orthodromically conducted sensory responses were detectable in the ulnar side of the middle finger and radial side of the ring finger stimulation with clinical improvement after the surgery, left median SNAP amplitudes were diminished postoperatively in antidromic response with the middle finger recording, and orthodromically recorded sensory response in the radial side of the middle finger. These findings could occur after an operative process such as neuroma excision, and microscope-assisted direct end to end neurorrhaphy.

In conclusion, we report a patient with a palmar digital neuropathy, with a rare anatomical variation of the median nerve. Also, usefulness of an orthodromic sensory conduction study was clarified to eliminate volume conducted response or co-activation of nearby nerves in the patient with selective involvement of palmar digital nerve. Knowledge of anatomic variation of common palmar digital nerves is essential, as it may be damaged during surgery or in trauma.

\section{CONFLICT OF INTEREST}

No potential conflict of interest relevant to this article was reported.

\section{AUTHOR CONTRIBUTION}

Conceptualization: Kwon HK. Methodology: Kwon HK, Park JW. Formal analysis: None. Funding acquisition: none. Project administration: none. Visualization: none. Writing - original draft: Noh JS. Writing - review and editing: Kwon HK. Approval of final manuscript: all authors.

\section{SUPPLEMENTARY MATERIALS}

Supplementary materials can be found via https://doi. org/10.5535/arm.2019.43.3.341. Table S1. Needle electromyography study. Fig. S1. Transverse ultrasonogram demonstrated no abnormal finding in the palm of left hand including a 3rd web space (A) and compression and swelling of median nerve with fluid collection at distal wrist crease (B and C). Arrows indicate fluid collection, and arrowheads indicate median nerve.

\section{REFERENCES}

1. Tian D, Fu M. Anatomic variation of the common palmar digital nerves and arteries. J Korean Neurosurg Soc 2015;57:219-20.

2. Zanette G, Lauriola MF, Tamburin S. An electrodiagnostic technique for assessing palmar proper digital nerves of the hand: Normative data and clinical application. Muscle Nerve 2015;52:972-80.

3. Zanette G, Lauriola MF, Tamburin S. Electrodiagnosis of lesions of median and ulnar nerve hand sensory branches: a case series. J Clin Neurophysiol 2016;33:454-7.

4. Henry BM, Zwinczewska H, Roy J, Vikse J, Ramakrishnan PK, Walocha JA, et al. The prevalence of anatomical variations of the median nerve in the carpal tunnel: a systematic review and meta-analysis. PLoS One 2015;10:e0136477.

5. Lanz U. Anatomical variations of the median nerve in the carpal tunnel. J Hand Surg Am 1977;2:44-53.

6. Schultz RJ, Endler PM, Huddleston HD. Anomalous median nerve and an anomalous muscle belly of the first lumbrical associated with carpal-tunnel syndrome. J Bone Joint Surg Am 1973;55:1744-6.

7. Goddard DH, Barnes CG, Berry H, Evans S. Measurement of nerve conduction: a comparison of orthodromic and antidromic methods. Clin Rheumatol 1983; 2:169-74.

8. Scholten RJ, Mink van der Molen A, Uitdehaag BM, Bouter LM, de Vet HC. Surgical treatment options for carpal tunnel syndrome. Cochrane Database Syst Rev 2007;(4):CD003905. 
9. Kwon HK, Lee HJ. Serial electrodiagnostic evaluation after carpal tunnel release. J Korean Acad Rehabil Med 2000;24:52-7.

10. Kim JY, Yoon JS, Kim SJ, Won SJ, Jeong JS. Carpal tun- nel syndrome: clinical, electrophysiological, and ultrasonographic ratio after surgery. Muscle Nerve 2012;45:183-8. 


\section{SUPPLEMENTARY MATERIALS}

Table S1. Needle electromyography study

\begin{tabular}{|c|c|c|c|c|c|c|c|}
\hline & \multirow{2}{*}{$\begin{array}{c}\text { Insertional } \\
\text { activity }\end{array}$} & \multirow{2}{*}{$\begin{array}{c}\text { Spontaneous } \\
\text { activity }\end{array}$} & \multicolumn{4}{|c|}{ Motor unit action potentials } & \multirow{2}{*}{ IP } \\
\hline & & & Normal & Polyphasia & Amplitude & Duration & \\
\hline Lt. brachioradialis & Normal & - & Normal & - & - & - & Full \\
\hline Lt. pronator teres & Normal & - & Normal & - & - & - & Full \\
\hline Lt. EDC & Normal & - & Normal & - & - & - & Full \\
\hline Lt. FDI & Normal & - & Normal & - & - & - & Full \\
\hline Lt. APB & Normal & - & - & - & Large $(8 \mathrm{mV})$ & Long & Reduced \\
\hline
\end{tabular}

IP, interference pattern; EDC, extensor digitorum communis; FDI, first dorsal interosseous; APB, abductor pollicis brevis.
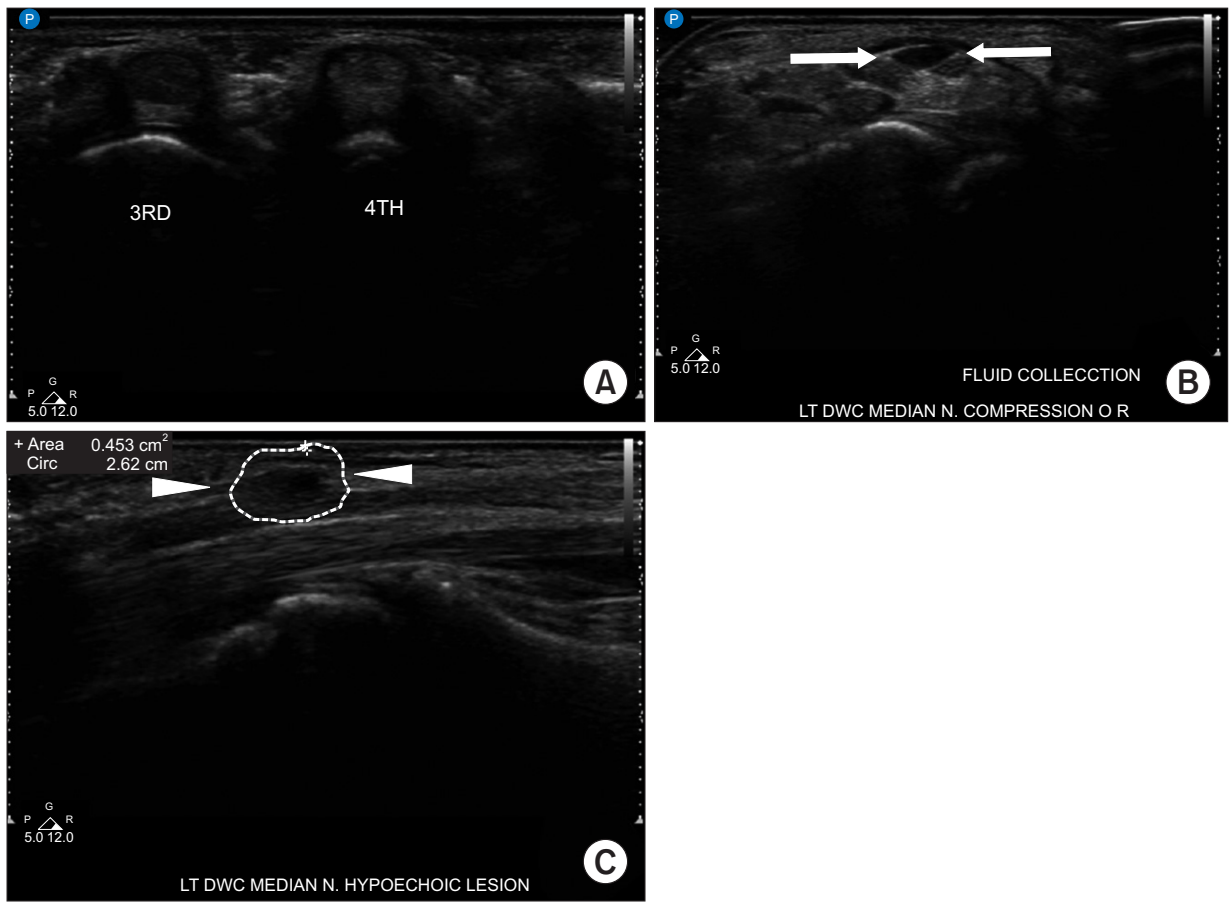

Fig. S1. Transverse ultrasonogram demonstrated no abnormal finding in the palm of left hand including a 3rd web space (A) and compression and swelling of median nerve with fluid collection at distal wrist crease (B and C). Arrows indicate fluid collection, and arrowheads indicate median nerve. 